\title{
Clinical significance and biological roles of small nucleolar RNAs in hepatocellular carcinoma (Review)
}

\author{
DILIP BARAL ${ }^{1}$, LONG WU $^{1}$, GAURAV KATWAL $^{1}$, XIONG YAN $^{1}$, YANFENG WANG $^{1}$ and QIFA YE $^{1,2}$ \\ ${ }^{1}$ Wuhan University, Zhongnan Hospital of Wuhan University, Institute of Hepatobiliary Diseases of Wuhan University, \\ Transplant Center of Wuhan University, Hubei Key Laboratory of Medical Technology on Transplantation, \\ Wuhan, Hubei $430071 ;{ }^{2}$ The Third Xiangya Hospital of Central South University, \\ Research Center of National Health Ministry on Transplantation Medicine \\ Engineering and Technology, Changsha, Hunan 410013, P.R. China
}

Received November 16, 2017; Accepted January 26, 2018

DOI: 10.3892/br.2018.1063

\begin{abstract}
Hepatocellular carcinoma (HCC) is among the most common and fatal cancers. It is a multistage and multifactorial carcinoma, in which a number of factors serve roles in its initiation and progression. Small nucleolar RNAs (snoRNAs), considered to serve a role in various cancers, have recently been identified to have significant contributions to HCC tumorigenesis. Recent studies suggest that snoRNAs have a critical role in the pathogenesis of HCC. Moreover, detailed studies have demonstrated that various snoRNAs are involved in a range of biological processes associated with HCC, including initiation, proliferation, tumor growth, the cell cycle, apoptosis and metastasis. In the present review, an overview of recent studies to date has been provided, focusing on the association of snoRNAs with HCC. Based on the findings, further studies focusing on the association of snoRNAs with HCC are required to verify the diagnostic and therapeutic capacities of snoRNAs in HCC.
\end{abstract}

\section{Contents}

1. Introduction

2. SnoRNAs

3. SNORD113-1

4. SNORD126

5. SNORD78

6. ACA11

Correspondence to: Dr YanFeng Wang, Wuhan University, Zhongnan Hospital of Wuhan University, Institute of Hepatobiliary Diseases of Wuhan University, Transplant Center of Wuhan University, Hubei Key Laboratory of Medical Technology on Transplantation, 169 East Lake Road, Wuchang District, Wuhan, Hubei 430071, P.R. China

E-mail: yanfengwang@whu.edu.cn

Key words: small nucleolar RNA, noncoding RNA, hepatocellular carcinoma, clinical significance, biological roles

\author{
7. SNORA47 \\ 8. SNORD76 \\ 9. Conclusion and future perspectives
}

\section{Introduction}

Hepatocellular carcinoma (HCC) is the most prevalent primary liver cancer, and is the third leading cause of cancer-related mortality worldwide and the second leading cause of cancer-related mortality in China and Southeast Asia (1). HCC is often detected at an advanced stage, and a lack of effective therapies for advanced stages contributes to its high rate of mortality $(2,3)$. HCC is a typical multistage disease, wherein a number of genes related to various cellular processes including cell cycle control, cell growth, apoptosis and cell migration are dysregulated (4). However, the molecular mechanisms of HCC tumorigenesis have not been fully elucidated to date. More in-depth knowledge of the molecular changes in HCC would aid to develop effective intervention strategies for the treatment of HCC.

As the name suggests, small nucleolar RNAs (snoRNAs) are located in the nucleolus. SnoRNAs are a class of non-coding RNAs of 60-300 nucleotides in length, responsible for post-transcriptional modification of other non-protein coding RNAs including ribosomal RNAs (rRNAs) and small nuclear RNAs (snRNAs) (5). SnoRNAs are among the most abundant groups of noncoding RNAs, with hundreds of them identified in a variety of organisms (6). SnoRNAs are divided into two families based on their structure and main function: Box C/D snoRNAs (SNORDs) and box H/ACA snoRNAs (SNORAs). SNORDs are responsible for 2'-O-ribose methylation, while the latter family guides pseudouridylation of nucleotides (7). As snoRNAs are involved in the process of post-transcriptional modifications of rRNAs, and hyperactive biogenesis of ribosomes is a common feature of cancer, it is proposed that dysregulation of snoRNAs may lead to cancer (8). Recent studies have indicated that snoRNAs may have significant influence on the development and progression of various cancers. Roles of various snoRNAs have been studied in cancers of the lung (9-12), colon and rectum (13), breast $(14,15)$, prostate (16), 
and in B and T cell lymphoma $(17,18)$, which has encouraged studies on the role of snoRNAs in HCC.

Therefore, the present review is focused on HCC-related snoRNAs with abnormal expression in tumor tissues and their mechanisms of action. Although few studies on snoRNAs in HCC have been published, understanding the unique role played by snoRNAs in HCC may be important in therapeutic decision-making. Given the critical roles of snoRNAs in the initiation and progression of other cancers $(10-13,16,18,19)$, it follows that researchers have become interested in the role of snoRNAs in HCC. In vitro and in vivo functional experiments in HCC cells have indicated that snoRNAs are involved in the regulation of various biological processes, including tumor cell proliferation, migration, apoptosis, the cell cycle, tumorigenesis and metastasis. SnoRNAs may also serve as diagnostic or prognostic biomarkers and therapeutic targets for HCC $(8,20-24)$. Interpreting the roles of snoRNAs in tumors may contribute to an improved understanding of the molecular mechanisms of HCC and assist in the development of novel therapeutic targets. In the present review, recent progress in research on the biological functions of snoRNAs in HCC is summarized, and the clinical implications of relevant snoRNAs as diagnostic or prognostic biomarkers and molecular therapeutic targets are evaluated.

A literature search using the PubMed (https://www.ncbi. nlm.nih.gov/pubmed) and Embase Library (https://www. embase.com/\#search) databases for papers published in English up to October 2017 was performed using the cross headings "snoRNA/small nucleolar RNA" and "hepatocellular carcinoma"; these were required to appear in the title or abstract, with all those articles lacking mention of the search terms in their title/abstract were excluded from the review. An overview of current findings regarding the clinical significance and biological roles of snoRNAs in the hepatocellular carcinoma was formulated from the articles obtained.

\section{SnoRNAs}

The roles of snoRNAs in various disease processes and oncogenesis have been previously studied $(6,7)$, but their role in $\mathrm{HCC}$ has been investigated only more recently. Thus, there are few studies that regard the roles of snoRNAs in HCC. Although the underlying mechanisms of HCC-related snoRNAs remain unidentified, the biological functions of snoRNAs have often been linked with certain signaling pathways $(7,22,24)$, and accompany apparent expression of the disease in liver cancer tissues. The dysregulated snoRNAs in HCC are projected to become novel biomarkers for diagnosis or the evaluation of therapeutic efficacy. The present review summarizes six well-documented snoRNAs in HCC: SNORD113-1 (21), SNORD126 (24), SNORD78 (8), ACA11 (22), SNORA47 (20) and SNORD76 (23). Among these snoRNAs, upregulated expression in $\mathrm{HCC}$ is considered to have an oncogenic function, while downregulated expression may exert tumor suppressive effects (Table I).

\section{SNORD113-1}

The SNORD113-1 gene is located at chromosome 14q32, and genomic alterations in $14 \mathrm{q} 32$ have been observed in HCC (25).
A recent study on the role of SNORD113-1 in HCC demonstrated that it was significantly downregulated in tumor tissues compared with non-tumor tissues; furthermore, a statistically significant association between low-level expression of SNORD113-1 and relapse-free survival was also observed, which signified that downregulation of SNORD113-1 was associated with the aggressiveness of HCC (21). However, the expression of SNORD113-1 was identified to have no significant correlation with clinicopathological features including age, sex or hepatitis B surface antigen ( $\mathrm{HBsAg}$ ). Hypermethylation of the putative promoter region of SNORD113-1, known as CpG, lead to a downregulation in the expression of SNORD113-1 in tumor cells compared with normal cells (21). Furthermore, it decreased tumorigenesis in vitro when studied in HCC cell lines, and in vivo in a xenograft nude mouse model (21). This decrease in tumorigenesis was identified to occur through induction of cell cycle arrest during cell proliferation rather than through apoptosis. Meanwhile, SNORD113-1 had no effect on the migration and invasion of the tumor cells. The transforming growth factor- $\beta$ and mitogen-activated protein kinase/extracellular signal-regulated kinase pathways were involved in this suppression of tumorigenesis by SNORD113-1 (21).

\section{SNORD126}

SNORD126 is located within the intron of its host gene, cyclin B1-interacting protein 1 (CCNB1IP1) on chromosome $14(24,26)$, and alteration in this chromosomal locus has been identified in HCC (27). A study performed by Fang et al (24) demonstrated that SNORD126 was upregulated to a high level in HCC and colorectal carcinoma compared with non-tumor tissues. Their study also indicated that SNORD126 expression was significantly correlated with Barcelona Clinic Liver Cancer (BCLC) stage in HCC patients. However, its expression was not associated with age, gender or alpha-fetoprotein (AFP) or HBsAg expression in HCC patients (24). Meanwhile, increased expression of SNORD126 was associated with shorter survival rate in the HCC patients. Using Huh-7 cell lines, it was also identified that SNORD126 overexpression increased cell growth and mediated resistance to cisplatin, etoposide and vinblastine (24). However, overexpression of SNORD126 did not significantly affect the migration of the Huh-7 cell lines. It was further observed that SNORD126 did not regulate the expression of its host gene CCNB1IP1 in HCC samples (24). In vitro and in vivo studies revealed that the phosphoinositide 3-kinase (PI3K)/protein kinase B (AKT) signaling pathway was involved, through the upregulation of fibroblast growth receptor 2, in facilitating the SNORD126-mediated malignancy (24).

\section{SNORD78}

SNORD78 is located at chromosome 1q25 (28). Amplification of this chromosomal region has been frequently reported in many solid tumors (29), including gastric carcinoma (30), non-small cell lung cancer $(31,32)$ and colorectal cancer (33). In a previous study, SNORD78 was identified to be upregulated in $47 \mathrm{HCC}$ surgical specimens compared 
Table I. HCC-related snoRNAs in the current review.

\begin{tabular}{lcccccc}
\hline snoRNA & $\begin{array}{c}\text { Chromosomal } \\
\text { location }\end{array}$ & Dysregulation & $\begin{array}{c}\text { Sample size, } \mathrm{n}, \\
\text { HCC/control }\end{array}$ & $\begin{array}{c}\text { Biological } \\
\text { roles }\end{array}$ & Author & Refs. \\
\hline SNORD 113-1 & $14 \mathrm{q} 32$ & Downregulated & $112 / 112$ & $\begin{array}{l}\text { Suppresses HCC cell growth } \\
\text { and proliferation; no effect on } \\
\text { migration or cell invasion. }\end{array}$ & Xu et al & (21)
\end{tabular}

SNORD126

14q11.2 Upregulated

$30 / 28$

SNORD78

$1 \mathrm{q} 25$

Upregulated

$47 / 47$

ACA11

$4 \mathrm{q} 16.3$

Upregulated

92/92

SNORA47

$5 \mathrm{q} 13.3$

Upregulated

$60 / 60$

SNORD76
Upregulated
Promotes HCC cell growth and promotes resistance to chemotherapy.

Promotes HCC cell proliferation, migration and invasion.

Promotes proliferation, in HCC cells.

Promotes HCC cell proliferation, migration, invasion and metastasis. invasion and migration

Fang et al

Ma et al

Wu et al

Promotes HCC development, Wu et al growth and invasion.

HCC, hepatocellular carcinoma; snoRNA, small nucleolar RNA.

with non-neoplastic liver tissues (8). This upregulation of SNORD78 was linked with the aggressive phenotype and poor prognosis of HCC. Furthermore, increased expression of SNORD78 was observed to be associated with decreased overall survival and recurrence-free survival rates of the patients with HCC (8). When studied in human HCC cell lines (Huh7, HCCLM9, Hep3B and SK-Hep1), SNORD78 had no significant effect on expression of its host gene, growth arrest specific 5 (GAS5). Furthermore, knockdown of SNORD78 in the SK-Hep1 cells lead to decreased cell proliferation, migration potential and invasion ability and also induced cell cycle arrest at G0/G1 phase while increasing cell apoptosis (8). This study provided insight into the association of SNORD78 with HCC, though the mechanism by which SNORD78 promotes HCC is yet to be fully elucidated. Further research on this may guide techniques for earlier diagnosis and improved therapeutic strategies in the future.

\section{ACA11}

The ACA11 gene is located on chromosome 4q16.3, which is established to be an amplified chromosomal segment in solid tumors, particularly HCC $(34,35)$. ACA11 is overexpressed in primary bladder, colon and esophageal cancers, which makes it a possible biomarker for these cancers (36). A previous study on 92 pairs of human HCC tissues and corresponding non-tumor tissues revealed that ACA11 was significantly increased in the HCC specimens compared with the non-tumor tissues (22). This upregulation was associated with histological grade, hepatitis B virus (HBV) infection, BCLC stage and portal vein tumor thrombus (PVTT). Furthermore, high-level ACA11 expression was associated with shorter overall survival and higher recurrence rates in the HCC patients (22). Downregulation of ACA11 in cell lines with high endogenous expression of ACA11, namely HCCLM9 and SK-Hep1 cells, resulted in reduced cell proliferation, migration ability and invasiveness (22). Accordingly, an in vivo study in nude mice identified reduced tumor and weight when ACA11 was downregulated (22). Furthermore, overexpression of ACA11 in Huh7 cells with low endogenous expression of ACA11 resulted in an increased level of cell proliferation, migration and invasion compared with controls, which was further supported by increased tumor weight in an in vivo study in nude mice (22). Collectively these findings indicated that ACA11 is associated with the tumorigenesis, aggressive behavior and prognosis of HCC. Additionally, through in vitro study, it was demonstrated that the PI3K/AKT pathway was activated during the ACA11-mediated effects in HCC (22). The PI3K/AKT pathway regulates cyclin D1 and epithelial-mesenchymal transition (EMT) markers, which are associated with cell proliferation, migration and invasion in tumors (37). With the knockdown of ACA11 in HCCLM9 and SK-Hep1 cells, 
the expression of epithelial markers such as claudin-1 was increased, while cyclin D1 and mesenchymal markers including fibronectin (FN1), neural (N)-cadherin and vimentin (VIM) were downregulated; the opposite result was observed when ACA11 was upregulated in those cell lines (22). This further suggests that ACA11 has an impact on HCC cell proliferation, invasion and migration.

\section{SNORA47}

SNORA47 is located at chromosomal region 5q13.3 (38), which is among the frequent locations of chromosomal gain in HCC (28). Significant upregulation of SNORA47 has been identified in a study of $60 \mathrm{HCC}$ tissues compared with normal specimens (20). The level of expression of SNORA47 was significantly correlated with intrahepatic metastasis, lymphatic invasion and Tumor-Node-Metastasis stage, while no association was identified with other clinicopathological variables including age, gender, size of the primary tumor, serum AFP, liver cirrhosis, PVTT or tumor differentiation. Furthermore, patients with high SNORA47 expression had significantly shorter overall survival rates and statistically higher recurrence rates than those with low expression of SNORA47 (20). Among human HCC cell lines (Hep3B, Huh-7, HCCLM9, SK-Hep1 and SMMC-7721), the expression of SNORA47 was identified to be relatively high in the SK-Hep1 and HCCLM9 cells (20). Knockdown of SNORA47 in the SK-Hep1 and HCCLM9 cell lines induced a decline in the proliferation of the cells, and the proportion of cells in early apoptosis was increased, thus implicating that knockdown of SNORA47 in those cell lines suppressed cell proliferation while increasing apoptosis. Furthermore, downregulation of SNORA47 in the SK-Hep1 and HCCML9 cell lines lead to reductions in the migration and invasion abilities of the cells (20). EMT serves a key role in the process of tumor metastasis and invasion $(39,40)$. In the aforementioned study, the knockdown of SNORA47 resulted in downregulation of zinc finger E-box binding homeobox 1, VIM, FN1 and $\mathrm{N}$-cadherin and an upregulation of epithelial (E)-cadherin, suggesting that SNORA47 induces the expression of these EMT markers (20). Taken together, it may be proposed that SNORA47 is not only upregulated in HCC but also affects tumorigenesis by inducing EMT.

\section{SNORD76}

SNORD76 is located within the third intron of the snoRNA host gene GAS5 at chromosome 1q25 (28). The chromosomal locus 1q25 is among the most frequently amplified chromosomal segments in solid tumors, particularly $\operatorname{HCC}(27,41)$. SNORD76 has been identified to be markedly upregulated (up to 3.5-fold) in HCC tissues in comparison to controls (23); furthermore, the expression of SNORD76 had substantial predictive significance for $\mathrm{HCC}$ in receiver operating characteristic curve analysis. It was proposed that SNORD76 may be used in clinical practice as a diagnostic marker for HCC (23), though further large-scale multicenter study is required prior to any clinical application. Nonetheless, high-level expression of SNORD76 was associated with histological grade, HBV infection, BCLC stage and PVTT in HCC patients (23).
Additionally, shorter overall survival rate was linked with the high-level expression of SNORD76. This suggests that SNORD76 may be a useful prognostic marker of HCC. In HCC cell lines, SK-Hep1 and Huh7, knockdown of SNORD76 caused arrest of the cell cycle at G0/G1 phase and increased apoptosis, while overexpression of SNORD76 significantly induced cell proliferation (23). An in vivo study in a xenograft nude mice model supported these findings, as there was decreased tumor growth when suppressing the expression of SNORD76 (23). Furthermore, the mesenchymal markers of EMT (vimentin, $\mathrm{N}$-cadherin and fibronectin) were induced while the epithelial markers of EMT (claudin-1 and E-cadherin) were reduced with the overexpression of SNORD76, whereas the opposite result occurred with knockdown of SNORD76 in vitro (23); this supports that SNORD76 induces the invasiveness of HCC via the EMT pathway. The $\mathrm{Wnt} / \beta$-catenin pathway, a major pathway involved in the tumorigenesis of HCC (19), was also implicated in the SNORD76-mediated effects on HCC (23).

\section{Conclusion and future perspectives}

In conclusion, there are limited studies on the role of snoRNAs in HCC. Thus, few studies to date have delineated the role of snoRNAs in HCC with regard to cancer initiation, progression, metastasis and recurrence, and treatment and prognosis. The dysregulation of snoRNA in HCC tissues is associated with these oncological aspects. To date, snoRNA in HCC has been studied through the use of patient tumor specimens, in addition to cell lines and xenograft mice models, and its potential role as a diagnostic and prognostic marker has been suggested. However, the invasiveness and costs associated with obtaining tissue specimens for diagnostic purposes may limit its usefulness in a clinical environment. Therefore, future studies should investigate whether or not snoRNAs are highly expressed in serum or other bodily fluids in patients with HCC. HCC is a multistage disease and various factors and molecules serve an interconnected role in its development and progression. It is critical to understand how snoRNAs interact with RNA, DNA and proteins and how aberrant crosstalk may be controlled in HCC. The majority of the previous studies concerning snoRNAs have been retrospective single-center analyses with a relatively small sample size. Thus, a multicenter prospective cohort study with a large sample is required to gain an improved understanding of the overt roles of snoRNAs in HCC in various populations and ethnic groups.

\section{Acknowledgements}

The authors would like to thank Dr Xiaoli Fan and Dr Zehong Fang for their help and support during the preparation of this article.

\section{Funding}

The present work was supported by the National Natural Science Foundation of China (grant no. 81570079).

\section{Availability of data and materials}

Not applicable. 


\section{Authors' contributions}

DB designed the review, searched the material, prepared and revised the draft; LW designed and revised the draft; GK searched the material and revised the draft; XY edited and revised the draft; YFW designed and supervised the project and revised the draft; QY designed and supervised the project. All authors read and approved the final draft.

\section{Ethics approval and consent to participate}

Not applicable.

\section{Consent for publication}

Not applicable.

\section{Competing interests}

The authors declare that they have no competing interests.

\section{References}

1. Torre LA, Bray F, Siegel RL, Ferlay J, Lortet-Tieulent J and Jemal A: Global cancer statistics, 2012. CA Cancer J Clin 65: 87-108, 2015

2. Poh Z, Shen L, Yang H-I, Seto WK, Wong VW, Lin CY, Goh BB, Chang PE, Chan HL, Yuen MF, et al: Real-world risk score for hepatocellular carcinoma (RWS-HCC): A clinically practical risk predictor for HCC in chronic hepatitis B. Gut 65: 887-888, 2016.

3. Lan T, Chang L, Wu L and Yuan Y: Downregulation of ZEB2-AS1 decreased tumor growth and metastasis in hepatocellular carcinoma. Mol Med Rep 14: 4606-4612, 2016.

4. Aravalli RN, Steer CJ and Cressman ENK: Molecular mechanisms of hepatocellular carcinoma. Hepatology 48: 2047-2063, 2008

5. Kiss T: Small nucleolar RNAs: An abundant group of noncoding RNAs with diverse cellular functions. Cell 109: 145-148, 2002.

6. Thorenoor N and Slaby O: Small nucleolar RNAs functioning and potential roles in cancer. Tumour Biol 36: 41-53, 2015.

7. Stepanov GA, Filippova JA, Komissarov AB, Kuligina EV, Richter VA and Semenov DV: Regulatory role of Small nucleolar RNAs in human diseases. Biomed Res Int 2015: 206849, 2015.

8. Ma P, Wang H, Han L, Jing W, Zhou X and Liu Z: Up-regulation of small nucleolar RNA 78 is correlated with aggressive phenotype and poor prognosis of hepatocellular carcinoma. Tumour Biol 37: $15753-15761,2016$.

9. Gao L, Ma J, Mannoor K, Guarnera MA, Shetty A, Zhan M, Xing L, Stass SA and Jiang F: Genome-wide small nucleolar RNA expression analysis of lung cancer by next-generation deep sequencing. Int J Cancer 136: E623-E629, 2015.

10. Mannoor K, Shen J, Liao J, Liu Z and Jiang F: Small nucleolar RNA signatures of lung tumor-initiating cells. Mol Cancer 13: 104, 2014.

11. Liao J, Yu L, Mei Y, Guarnera M, Shen J, Li R, Liu Z and Jiang F: Small nucleolar RNA signatures as biomarkers for non-small-cell lung cancer. Mol Cancer 9: 198, 2010.

12. Mei Y-P, Liao J-P, Shen J, Yu L, Liu BL, Liu L, Li RY, Ji L, Dorsey SG, Jiang ZR, et al: Small nucleolar RNA 42 acts as an oncogene in lung tumorigenesis. Oncogene 31: 2794-2804, 2012.

13. Okugawa Y, Toiyama Y, Toden S, Mitoma H, Nagasaka T, Tanaka K, Inoue Y, Kusunoki M, Boland CR and Goel A: Clinical significance of SNORA42 as an oncogene and a prognostic biomarker in colorectal cancer. Gut 66: 107-117, 2017.

14. Su H, Xu T, Ganapathy S, Shadfan M, Long M, Huang TH, Thompson I and Yuan ZM: Elevated snoRNA biogenesis is essential in breast cancer. Oncogene 33: 1348-1358, 2014.

15. Dong X-Y, Guo P, Boyd J, Sun X, Li Q, Zhou W and Dong JT: Implication of snoRNA U50 in human breast cancer. J Genet Genomics 36: 447-454, 2009

16. Dong XY, Rodriguez C, Guo P, Sun X, Talbot JT, Zhou W, Petros J, Li Q, Vessella RL, Kibel AS, et al: SnoRNA U50 is a candidate tumor-suppressor gene at $6 \mathrm{q} 14.3$ with a mutation associated with clinically significant prostate cancer. Hum Mol Genet 17: 1031-1042, 2008 .
17. Tanaka R, Satoh H, Moriyama M, Satoh K, Morishita Y, Yoshida S, Watanabe T, Nakamura Y and Mori S: Intronic U50 small-nucleolar-RNA (snoRNA) host gene of no protein-coding potential is mapped at the chromosome breakpoint $\mathrm{t}(3 ; 6)(\mathrm{q} 27 ; \mathrm{q} 15)$ of human B-cell lymphoma. Genes Cells 5: 277-287, 2000.

18. Valleron W, Ysebaert L, Berquet L, Fataccioli V, Quelen C, Martin A, Parrens M, Lamant L, de Leval L, Gisselbrecht C, et al: Small nucleolar RNA expression profiling identifies potential prognostic markers in peripheral T-cell lymphoma. Blood 120: 3997-4005, 2012.

19. Giakoustidis A, Giakoustidis D, Mudan S, Sklavos A and Williams R: Molecular signalling in hepatocellular carcinoma: Role of and crosstalk among WNT/ß-catenin, Sonic Hedgehog, Notch and Dickkopf-1. Can J Gastroenterol Hepatol 29: 209-217, 2015.

20. Li G, He Y, Liu X, Zheng Z, Zhang M, Qin F and Lan X: Small nucleolar RNA 47 promotes tumorigenesis by regulating EMT markers in hepatocellular carcinoma. Minerva Med 108: 396-404, 2017.

21. Xu G, Yang F, Ding C-L, Zhao LJ, Ren H, Zhao P, Wang W and Qi ZT: Small nucleolar RNA 113-1 suppresses tumorigenesis in hepatocellular carcinoma. Mol Cancer 13: 216, 2014.

22. Wu L, Zheng J, Chen P, Liu Q and Yuan Y: Small nucleolar RNA ACA11 promotes proliferation, migration and invasion in hepatocellular carcinoma by targeting the PI3K/AKT signaling pathway. Biomed Pharmacother 90: 705-712, 2017.

23. Wu L, Chang L, Wang H, Ma W, Peng Q and Yuan Y: Clinical significance of C/D box small nucleolar RNA U76 as an oncogene and a prognostic biomarker in hepatocellular carcinoma. Clin Res Hepatol Gastroenterol: May 31, 2017 (Epub ahead of print).

24. Fang X, Yang D, Luo H, Wu S, Dong W, Xiao J, Yuan S, Ni A, Zhang KJ, Liu XY, et al: SNORD126 promotes HCC and CRC cell growth by activating the PI3K-AKT pathway through FGFR2. J Mol Cell Biol 9: 243-255, 2017.

25. Piao Z, Park C, Park JH and Kim H: Allelotype analysis of hepatocellular carcinoma. Int J Cancer 75: 29-33, 1998.

26. Yang JH, Zhang XC, Huang ZP, Zhou H, Huang MB, Zhang S, Chen YQ and Qu LH: snoSeeker: An advanced computational package for screening of guide and orphan snoRNA genes in the human genome. Nucleic Acids Res 34: 5112-5123, 2006.

27. Katoh H, Shibata T, Kokubu A, Ojima H, Loukopoulos P, Kanai Y, Kosuge T, Fukayama M, Kondo T, Sakamoto M, et al: Genetic profile of hepatocellular carcinoma revealed by array-based comparative genomic hybridization: Identification of genetic indicators to predict patient outcome. J Hepatol 43: 863-874, 2005

28. Yoshihama M, Nakao A and Kenmochi N: snOPY: A small nucleolar RNA orthological gene database. BMC Res Notes 6: 426, 2013

29. Makarova JA, Ivanova SM, Tonevitsky AG and Grigoriev AI: New functions of small nucleolar RNAs. Biochemistry (Mosc) 78: 638-650, 2013.

30. Sun M, Jin FY, Xia R, Kong R, Li JH, Xu TP, Liu YW, Zhang EB, Liu XH and De W: Decreased expression of long noncoding RNA GAS5 indicates a poor prognosis and promotes cell proliferation in gastric cancer. BMC Cancer 14: 319, 2014.

31. Shi X, Sun M, Liu H, Yao Y, Kong R, Chen F and Song Y: A critical role for the long non-coding RNA GAS5 in proliferation and apoptosis in non-small-cell lung cancer. Mol Carcinog 54 (Suppl 1): E1-E12, 2015.

32. Zheng D, Zhang J, Ni J, Luo J, Wang J, Tang L, Zhang L, Wang L, $\mathrm{Xu}$ J, Su B, et al: Small nucleolar RNA 78 promotes the tumorigenesis in non-small cell lung cancer. J Exp Clin Cancer Res 34: $49,2015$.

33. Yin D, He X, Zhang E, Kong R, De W and Zhang Z: Long noncoding RNA GAS5 affects cell proliferation and predicts a poor prognosis in patients with colorectal cancer. Med Oncol 31: 253,2014

34. Zhou P, Wu LL, Wu KM, Jiang W, Li JD, Zhou LD, Li XY, Chang S, Huang Y, Tan H, et al: Overexpression of MMSET is correlation with poor prognosis in hepatocellular carcinoma. Pathol Oncol Res 19: 303-309, 2013

35. Yu F, Shen XY, Fan L and Yu ZC: Genome-wide analysis of genetic variations assisted by Ingenuity Pathway Analysis to comprehensively investigate potential genetic targets associated with the progression of hepatocellular carcinoma. Eur Rev Med Pharmacol Sci 18: 2102-2108, 2014.

36. Chu L, Su MY, Maggi LB Jr, Lu L, Mullins C, Crosby S, Huang G, Chng WJ, Vij R and Tomasson MH: Multiple myeloma-associated chromosomal translocation activates orphan snoRNA ACA11 to suppress oxidative stress. J Clin Invest 122: 2793-2806, 2012. 
37. Liu L, He YZ, Dong PP, et al: Protein tyrosine phosphatase PTP4A1 promotes proliferation and transition in intrahepatic cholangiocarcinoma via the PI3K/AKT pathway. Oncotarget 7 : 75210-75220, 2016.

38. Lestrade L and Weber MJ: snoRNA-LBME-db, a comprehensive database of human H/ACA and C/D box snoRNAs. Nucleic Acids Res 34: D158-D162, 2006.

39. Kalluri R and Weinberg RA: The basics of epithelial-mesenchymal transition. J Clin Invest 119: 1420-1428, 2009.

40. Fagoonee S, Famulari ES, Silengo L, Camussi G and Altruda F: Prospects for Adult Stem Cells in the Treatment of Liver Diseases. Stem Cells Dev 25: 1471-1482, 2016.
41. Leung TH-Y, Wong N, Lai PB-S, Chan A, To KF, Liew CT, Lau WY and Johnson PJ: Identification of four distinct regions of allelic imbalances on chromosome 1 by the combined comparative genomic hybridization and microsatellite analysis on hepatocellular carcinoma. Mod Pathol 15: 1213-1220, 2002.

(i) $(9$ This work is licensed under a Creative Common Attribution-NonCommercial-NoDerivatives 4.0 International (CC BY-NC-ND 4.0) License. 